\title{
PRODUTIVIDADE E USO DE ITENS LEXICAIS: 0 CONTINUUM FALA/ESCRITA CIENTÍFICA
}

\author{
Vânia Lisbôa da Silveira Guedes (UFRJ) \\ Maria de Fatima Sousa de Oliveira Barbosa (UFRJ) \\ Maria Cecilia de Magalhães Mollica (UFRJ)
}

Resumo: Este estudo investiga a alternância e sistematicidade no uso de estruturas linguísticas na fala e na escrita científica em português do Brasil. Objetiva desenvolver uma análise comparativa léxico-morfológica de corpora e mapear o continuum fala/escrita científica ao analisar o uso de nominalizações, identificando o grau de carga semântica das estruturas em tela e de estratégias de polidez. Contribui assim para o fortalecimento da base teórica e empírica da análise de domínio na Organização do Conhecimento, sobretudo no que se relaciona ao desenvolvimento de sistemas precisos de indexação e recuperação da informação. A hipótese é a de que na fala/escrita, estabelece-se uma relação entre empregos de formas sufixais, sua produtividade e frequência de uso referente às especificidades da linguagem científica. Parte-se de pressupostos da análise crítica do discurso, da teoria dos gêneros discursivos, da pragmática, da indexação e da bibliometria. São analisados artigos sobre Vinicultura e um corpus sobre Química Geral, selecionados respectivamente na Scientific Electronic Library Online e na base de dados do Projeto Norma Linguística Urbana Culta e processados pelo Software RankWords2.0. Na fala, os resultados apontam a predominância de nominalizações em -ção, seguida, em ordem decrescente de frequência das nominalizações em-ncia e-agem; atestam ainda a ausência de nominalizações em -mento. Já na escrita, os achados revelam predominância de nominalizações em -ção, seguida de-mento, -ncia e-gem. Percebe-se que, na fala, a curva decrescente é bem acentuada, enquanto que, na escrita, a curva é suave. Pela variável frequência pode-se notar a relevância da nominalização como estratégia de polidez. Além disso, os resultados revelam a regularidade no uso de nominalizações com o sufixo -ção no campo semântico da Química Geral e da Vinicultura, a despeito de tratar de área especializada que exige rigorosamente terminologia especializada e com léxico restrito em relação ao campo de conhecimento de Química.

Palavras-chaves: Análise crítica do discurso, Escrita científica, Nominalização, Estratégia de polidez, Gênero acadêmico. 
Abstract: This study investigates the alternance and systematicity in the use of linguistic structures in speech and scientific writing in Brazilian Portuguese. The study aims to develop a lexical-morphological comparative analysis of corpora and to map the continuum speech/ writing from the use of nominalizations analysis, identifying the degree of semantic load of the linguistic structures and their politeness strategies. Thus the study contributes to the strengthening of the theoretical and empirical basis of the domain analysis in the Knowledge Organization, especially to the development of the subject indexing and information retrieval systems. The hypothesis is that the speech/writing continuum establishes a relationship between suffixes, productivity and use frequency related to the specificities of the scientific language. The theoretical framework used was the critical discourse analysis, genre theory, pragmatics, indexing and bibliometrics. To identify those lexico-morphological features in such terminology, two papers about the wine industry and a corpus of General Chemistry discourse were selected respectively in the Scientific Electronic Library Online and Database Standard Language Project Urban Cultured, processed by software RankWords2.0. In speech, the results indicate the predominance of nominalizations in--ção, followed in decreasing order of frequency by the nominalizations in -ncia and -agem; the result attest the absence of nominalizations in -mento. In the writing, the findings reveal a predominance of nominalizations in -ção, followed by -mento, -ncia e-agem. It is noticed that in speech the decreasing curve is very steep, whereas in writing the curve is smooth. By the frequency variable the relevance of the nominalization as politeness strategy is noted. Furthermore, the results reveal the regularity in the use of nominalizations with suffix -ção in the semantic field of General Chemistry and Wine Industry, in despite of dealing with specialized area that requires specialized and strictly restricted lexicon terminology in relation to the field of knowledge Chemistry.

Keywords: Critical discourse analysis. Scientific writing, Lexicalgrammatical constructions, Distancing strategies, Research genre.

\section{INTRODUÇÃO}

Esse estudo apresenta resultados de pesquisas em andamento, ligadas aos projetos de pós-doutoramento 
Análise da regularidade na produtividade de estruturas linguísticas no discurso científico: o continuum fala e escrita científica, no âmbito do Programa de Pós-graduação em Linguística da Universidade Federal do Rio de Janeiro e ao Projeto Organização e apropriação do conhecimento no âmbito pragmático-discursivo, sintático e cognitivo da linguagem $^{1}$, vinculado ao Programa de Pós-graduação em Ciência da Informação do Instituto Brasileiro de Informação em Ciência e Tecnologia, do Ministério de Ciência e Tecnologia, em convênio com a Universidade Federal do Rio de Janeiro. O estudo visa a investigar a alternância e sistematicidade do uso de nominalizações deverbais na fala e na escrita científica em língua portuguesa. Essas construções se caracterizam como marcadores de impessoalização e exercem função de índice na escrita de gênero acadêmico, o que demonstra a importância da análise léxico-gramatical de padrões linguísticos específicos para o conhecimento da escrita de textos na comunicação cientifica . A pesquisa objetiva (a) desenvolver uma análise comparativa léxicomorfológica de corpora e (b) identificar distintos graus de carga semântica de uso de estratégias de polidez pelo mapeamento do continuum fala/escrita do texto científico.

1 Os referidos projetos estão sendo empreendidos por Vânia Lisbôa da Silveira Guedes e Maria de Fatima Sousa de Oliveira Barbosa, ambos sob a orientação da professora Maria Cecilia de Magalhães Mollica. 
A motivação para o estudo prende-se às seguintes premissas: (i) intensificação de pesquisas com o propósito de ampliação de fundamentos teóricos e metodológicos na fronteira entre a Ciência da Informação e a Linguística para a identificação do grau de informatividade de itens lexicais na escrita científica; (ii) investigação da frequência de uso de itens lexicais como marca de impessoalização e polidez na comunicação científica.

A estrutura deste artigo se compõe das seguintes seções. Enquanto a primeira apresenta uma visão panorâmica da investigação, a segunda seção lança seus objetivos gerais e específicos. Os pressupostos teóricos acham-se resumidos na terceira seção. A metodologia descreve os critérios utilizados para a constituição da amostra e as etapas do procedimento de análise dos dados. Na seção 5, são apresentados e discutidos os resultados, utilizando-se tabelas e gráficos para a sintetização e a confrontação dos padrões importantes encontrados. As considerações finais encerram o texto, seguidas das referências ligadas aos documentos citados.

\section{OBJETIVOS}

O objetivo geral deste estudo consiste em investigar comparativamente a produtividade de estruturas linguísticas, com graus distintos de informatividade e denotadores de 
estratégia de polidez na fala e na escrita científica em língua portuguesa nas Ciências Químicas. Objetivos específicos são elencados resumidamente como se segue.

a) investigar a alternância e sistematicidade no uso de padrões de nominalizações deverbais em -ção, mento, -ncia e-agem., na fala e na escrita científica em língua portuguesa;

b) contribuir para as abordagens teóricas e descritivas da indexação de assunto na Ciência da Informação (CI);

c) apontar a tipificação das estratégias de polidez na comunicação científica;

d) intensificar pontos de interface entre a Teoria de Gêneros no âmbito da Análise Crítica do Discurso na Linguística e a Organização do Conhecimento na $\mathrm{Cl}$.

\section{PRESSUPOSTOS TEÓRICOS}

Como fundamentação teórico-metodológica, utilizaramse referenciais importantes e atualizados ligados aos seguintes campos do conhecimento: (i) organização do conhecimento, (ii) análise de gêneros discursivos e Análise Crítica do Discurso, (iii) nominalização, polidez e comunicação científica e (iv) indexação e recuperação da informação no âmbito da Bibliometria na Ciência da Informação. Foram destacados os estudos de Swales, Bazerman, Hylland, Andersen e 
Basílio que discorrem sobre fenômenos inerentes à escrita científica. Na área da Bibliometria, foram discutidas as leis de Zipf e o Ponto de Transição de Goffman como indicadores bibliométricos da região de concentração de palavras com alta carga semântica.

A Organização do Conhecimento (OC), de acordo com Dahlberg (2006), é a ciência que estuda a estruturação e o arranjo sistemático de unidades de um domínio semântico, entendidos como conceitos, considerando-Ihes os elementos e as características inerentes. Assim, para a OC, em um domínio específico, os conceitos enquanto unidades de conhecimento devem ser relacionados e representados sistematicamente, com a finalidade de estruturar termos e acepções para fins de representação, recuperação e comunicação da informação e do conhecimento. As diversas formas de estruturar o conhecimento são expressas nos Sistemas de Organização do Conhecimento (SOC) que, por sua vez, são sempre elaborados intencionalmente, estruturados a partir de pontos de vista específicos, permeados por aspectos culturais, intelectuais e linguísticos, particulares de uma comunidade de discurso determinada.

Embora em relação à elaboração dos diferentes SOC haja também uma corrente cognitiva que preconiza que a mente humana naturalmente organiza e classifica o conhecimento, 
Ørom (2003) defende a ideia de que não há relações autoevidentes ou universais, pois elas são sempre intencionais e influenciadas pelo contexto sociocultural. Os sistemas de organização do conhecimento envolvem a maneira pela qual determinado campo é conceituado e representado sistematicamente. De acordo com Soergel et al (2004), nos atuais e correntes SOC, há algumas limitações, tais como:

a) falta de abstração conceitual: os sistemas não refletem a compreensão de mundo dos seres humanos no que diz respeito ao sentido e à linguagem;

b) cobertura semântica limitada: conjunto restrito de relações conceituais, predominando as relações hierárquicas e/ou associativas que não são suficientes para a realização de pesquisas a partir de inferências;

c) falta de consistência: estrutura semântica interna irregular, imprevisível e por vezes ambígua, devido à ausência de relações semânticas bem estabelecidas.

Em suma, para Hjørland \& Albrechtsen (1995) e Ørom (2003), a construção dos sistemas de organização e representação do conhecimento deve considerar as possíveis garantias necessárias para a validação de tais sistemas, baseados na terminologia adotada pela comunidade discursiva, de forma a viabilizar a comunicação científica. 
Andersen (2008), ao aportar na interface da Ciência da Informação com a Linguística, salienta que os indivíduos e as sociedades humanas têm organizado e transmitido conhecimentos, significados e experiências por meios particulares de comunicação. As formas de comunicação são parte inerente da organização de qualquer cultura porque estruturam e sustentam significados institucionalizados na sociedade. O autor infere que a variedade de formas de comunicação e atividades científicas podem ser concebida como gênero. A teoria dos gêneros revela, portanto, como as atividades humanas e as produções discursivas são importantes fatores de organização da comunicação e do conhecimento. Bazerman (1998) sustenta que a teoria dos gêneros nos auxilia a navegar pelos mundos complexos da comunicação escrita e das atividades simbólicas. Andersen (2008) acrescenta que ela amplia nossa compreensão sobre como o conhecimento é organizado e comunicado em um discurso registrado. O autor enfatiza que as atividades humanas que envolvem o uso de textos se tornam tipificadas em consequência da produção e uso de discursos registrados em diferentes comunidades.

Já Hjørland (2002) defende que a análise de gêneros é um componente natural da Análise de Domínio no âmbito da 
Organização do Conhecimento na $\mathrm{Cl}$. Note-se que Andersen (2008) também esclarece que reconhecer um tipo de texto supõe o reconhecimento de uma situação comunicativa particular. Quanto mais sabemos acerca de uma atividade comunicativa na qual estamos envolvidos, mais passamos a compreender e a usar os textos produzidos por essa atividade. Textos e contextos estão em interação dinâmica com as regras de produção e leitura, assim como os modos de atuação discursiva caracterizam uma comunidade discursiva.

Assim, na perspectiva da Teoria do Gênero na Análise Crítica do Discurso, tendências recentes têm demonstrado atenção especial aos gêneros científicos. Nesse cenário, caracteriza-se a escrita científica como explícita, lexicalmente densa, impessoal, nominalizada, cautelosa, intertextual e referenciada (cf. Swales, 1990; Hyland, 2009; Eggins, 2004).

A comunicação científica em um campo específico do conhecimento é operada pelas formas distintas de comunicação utilizadas pelos cientistas que pertencem a uma comunidade específica de discurso. Na comunicação científica, Hyland (2009) afirma que os pesquisadores buscam encaixar sua escrita em um mundo social particular, por meio de um discurso aprovado por seus pares. Nesse sentido, Hyland \& Salanger-Meyer (2008) defendem que os textos 
são socialmente produzidos em comunidades particulares e dependem dessas comunidades para a obtenção de sentido.

Méndez Rodríguez \& Moreiro González (1999) destacam que a comunicação científica apresenta estrutura linguística própria e se estabelece em linguagem natural. Segundo os autores, o discurso científico é identificado pelas as seguintes características fundamentais: recepção e emissão qualificada (competência), vocabulário especializado, organização estrutural útil à ciência, modelo lógico-formal, determinação mais sistemática que a linguagem comum.

Ziman (1969) entende que a comunicação é essencial para a pesquisa científica. Ele considera que os resultados de pesquisa somente se tornam completamente científicos quando publicados. Enfatiza, ainda, que a invenção de um mecanismo para a publicação sistemática de fragmentos de trabalhos científicos pode ter sido o evento principal na história da ciência moderna.

Nesse cenário, Swales (1990) ressalta que o artigo científico emergiu, ainda que embrionariamente, com o estabelecimento do primeiro periódico cientifico intitulado The Philosophical Transactions of the Royal Society of London, em março de 1665, opinião compartilhada por Bazerman (2006) quando menciona que o primeiro artigo científico 
publicado no Philosophical originou-se da correspondência trocada entre o alemão Henry Oldenburg, então secretário da Royal Society of London desde 1662, e outros pesquisadores. Na função de secretário, sua correspondência foi intensificada e utilizada para a edição de textos publicados nesse periódico. O Philosophical Transactions of the Royal Society of London junto com o Journal des Savants, editado na França em 1665, são considerados os primeiros periódicos científicos que surgiram. (Guedes, 2010).

Sob a perspectiva da análise do gênero discursivo artigo de periódico científico, com propósito comunicativo, Guedes (2010), ao estabelecer a interface entre a Linguística e a Ciência da Informação, apresenta um estudo sobre o nível léxico-morfológico da língua focalizando em especial a nominalização deverbal, presente na escrita científica na área de indústria de vinhos. Vale assinalar que a nominalização, segundo Basílio (2007, p.77), "refere-se ao conjunto de processos que formam substantivos a partir de adjetivos e, sobretudo, de verbos". Analisando os aspectos discursivos, a autora destaca (i) as funções envolvidas com a expressão de atitudes subjetivas em relação ao objeto do enunciado e (ii) as funções associadas à adequação de itens lexicais ao gênero de discurso e à construção do 
enunciado. A autora esclarece que a "função semântica da nominalização é a de denominação", possibilitando a referência a um processo verbal, bem como a um evento, uma ação, um estado (Basílio, 2007, p. 83).

A Teoria da Polidez apresentada por Brown e Levinson (1978; 1987) tem sido usada para explicar uma dos principais objetivos dos atores sociais, o de atender aos desejos de face mantendo o território respeitado. Essa teoria se apoia nos estudos de Goffman (1976) que assegura que a face é o que se tem de mais pessoal, o centro de segurança e prazer. Entretanto, a face que se apresenta é apenas um empréstimo feito pela sociedade e pode-se perdê-la se não se fizer por merecê-la, daí o sentido de coconstrução na interação.

Os indivíduos vivem em constante interação social, seja face a face, seja por correspondência epistolar, canal de comunicação utilizado intensivamente em passado recente quando as tecnologias ainda não faziam parte do cotidiano midiático. Na interação, formal ou informal, os indivíduos tendem a assumir um padrão constituído por atos verbais e não verbais, os quais expressam sua visão da situação e da avaliação que fazem dos participantes e de si mesmo (Goffman, 1976). Os atos verbais e não verbais constantemente ameaçam a face de uma ou de outra das quatro faces envolvidas na 
interação, duas do ouvinte e duas do falante. Quando uma das faces é ameaçada, têm-se os Atos de Ameaça à Face (AAF), do inglês Face Threatening Act (FTA), apresentados por Brown e Levinson (1978). Pressupostamente, é interessante que todos os participantes trabalhem para que tanto os desejos de face do self quanto do outro sejam mantidos. Para isso, fazse uso de estratégias de polidez para manter a interação em harmonia. Nesse sentido, concordamos com Bakhtin (1992) quando afirma que

[...] Toda palavra comporta duas faces. Ela é determinada tanto pelo fato de que precede de alguém, como pelo fato de que se dirige para alguém. Ela constitui justamente o produto da interação do locutor e do ouvinte. Toda palavra serve de expressão a um em relação ao outro (BAKHTIN, 1992[1979] p. 99).

Neste trabalho, são observadas estruturas de nominalizações utilizadas pelos autores para imprimirem polidez na comunicação científica, evitando FTA entre os pares. Discutiremos a noção de polidez exigida entre atores sociais em seus atos interacionais. A interação na comunicação científica tem sido estudada por alguns autores, dentre os quais citamos Gil-Salom, L. \& SolerMonreal C. (2009), Hyland (2009; 2008) e Hyland \& SalengerMeyer (2008). 
A Bibliometria na Ciência da Informação segundo Miranda Pao (1989) refere-se à área do conhecimento que utiliza métodos matemáticos e estatísticos, com o objetivo de analisar os processos de comunicação escrita. Okubo (1997) afirma que as abordagens bibliométricas são baseadas na noção de que a produção de conhecimento é a essência da pesquisa científica e, nesse contexto, a literatura científica é a manifestação constituinte do conhecimento. Okubo esclarece que os dados e indicadores bibliométricos podem servir como ferramentas ao menos para a descrição e expressão de questões que surgem no mundo da Ciência. As principais leis bibliométricas são: Lei de Bradford, (produtividade de periódicos), Lei de Lotka (produtividade científica de autores) e Leis de Zipf (frequência de palavras).

George Kingsley Zipf (1902-1950), linguista, pesquisador e professor de Filologia da Universidade de Harvard, durante a primeira metade do Século XX, observou certas regularidades na estrutura linguística de textos. Na análise de frequência de ocorrência de palavras em um texto, enunciou duas leis, a Primeira e a Segunda Leis de Zipf. A primeira está relacionada às palavras de alta frequência e a segunda, às de baixa frequência.

Goffman, a partir da segunda lei de Zipf modificada por Booth, admitiu existir uma região na lista de palavras, 
localizada entre as palavras de alta frequência e as de baixa frequência, a que denominou de "região de transição", com probabilidade de concentrar as palavras de alto conteúdo semântico. Para se chegar a essa região, ele desenvolveu uma fórmula denominada de Ponto de Transição (T) de Goffman, representada matematicamente pela expressão

Onde:

$\mathrm{n}$ representa o ponto $\mathrm{T}$;

$\mathrm{I}_{1}$ é o número de palavras que tem frequência 1;

8 é uma constante derivada da língua inglesa;

2 é um número matemático da fórmula de Baskara, para resolução de equações de 2 o grau.

Ao verificar a hipótese de que a Região de Transição de Goffman reúne as palavras de maior conteúdo semântico, Pao (1978, 1989) delimitou a região projetando para baixo o mesmo número de palavras que ocorreu acima da frequência que corresponde ao Ponto T. Nessa região, foram excluídas as palavras sem conteúdo semântico, tais como: preposições, artigos, conjunções, pronomes e verbos auxiliares. Ao analisar os resultados obtidos, a autora conclui que eles foram satisfatórios para os textos analisados e parecem indicar que a seleção automática de termos de indexação, a partir de uma lista de frequência de 
ocorrência de palavras, é uma perspectiva promissora para a indexação automática.

É importante destacar que as ideias lançadas por Zipf fundamentam a moderna Linguística Quantitativa, no entanto, sua influência não se restringe a essa área, sendo também observada em outros domínios do conhecimento.

\section{METODOLOGIA}

Foram selecionados dois artigos sobre Vinicultura extraídos aleatoriamente da Scientific Electronic Library OnlinE e uma aula sobre Química Geral na base de dados do Projeto Norma Linguística Urbana Culta (NURC) da UFRJ. Segue o quadro 1 com a referência dos artigos e da aula.

T1: BEHRENS, Jorge H., SILVA, Maria Aparecida A. P. da, \& WAKELING, Ian N. (1999). Avaliação da aceitação de vinhos brancos varietais brasileiros através de testes sensoriais afetivos e técnica multivariada de mapa de preferência interno. Food Science and Technology (Campinas), 19(2), 214-220. Disponível em: <http:// www.scielo.br/scielo.php?script=sci_arttext\&pid=S010120611999000200011\&lng=en\&nrm=iso>. Acesso: 01 Julho 2013

T2: RIZZON, Luiz Antenor, MIELE, Alberto, \& MENEGUZZO, Júlio. (1999). Efeito da relação das fases líquida e sólida da uva na composição química e na característica sensorial do vinho Cabernet. Food Science and Technology (Campinas), 19(3), 424-428. Acesso: 01 Julho 2013. Disponível em: http://www.scielo.br/scielo. php?script=sci_arttext\&pid=S0101-20611999000300023\&lng=en \&tlng=pt.10.1590/S0101-20611999000300023 
T3: Aula Elocução Formal (EF). Aula de química para o terceiro científico. (Inquérito 251) do Projeto Norma Linguística Urbana Culta - RJ. Disponível em: http://www.letras.ufri.br/nurc-ri/Acesso: 01 Julho 2013 Quadro 1: Amostra

O experimento foi realizado cumprindo as etapas descritas a seguir.

- Delimitação da amostra - foram selecionadas aleatoriamente dois artigos sobre Vinicultura selecionados na Scientific Electronic Library OnlinE e um corpus sobre aula de Química Geral na base de dados do Projeto NURC;

- contagem das palavras - foi utilizado o software contador de palavras Rank Words 2.0;

- listagem e ordenação das palavras - o software produziu uma listagem, para cada corpus, em 3 colunas assim distribuídas: i) palavras, ii) frequência em ordem decrescente de ocorrência e iii) rank das palavras;

- cálculos matemáticos e estatísticos - os dados foram plotados em planilhas utilizando-se o Software Microsoft Office Excel 2003 e foram obtidos os seguintes produtos: total de palavras, total de palavras distintas, ranking de nominalizações deverbais;

- identificação dos padrões de nominalizações - foram investigados os seguintes padrões: (i) [X] $\mathrm{X} \rightarrow[\mathrm{X}] \vee$-ção ] 
$N$, (ii) $[X] \vee \rightarrow[[X] \vee-$ mento $] N$, (iii) $[X] \vee \rightarrow[$ [X] $\vee$-ncia ] N;

- cálculo da produtividade das nominalizações - foi calculada a produtividade relativa de cada padrão de nominalizações deverbais, considerando-se a frequência relativa das estruturas nominalizadas;

- controle de formas no singular e plural - foram consideradas as frequências de nominalizações somando-se suas formas no singular e no plural;

- aplicação da fórmula do Ponto de Transição (T) de Goffman - foi aplicada a fórmula com o propósito de identificação da região de transição, onde, segundo Goffman, encontram-se as palavras de alto conteúdo semântico;

- investigação das nominalizações - foram investigadas as nominalizações deverbais que se situam na região de concentração de palavras de alto conteúdo semântico;

- estratégia de polidez - verificação do uso de nominalizações com graus distintos de relevância como estratégia de polidez na comunicação científica;

- confrontação dos dados - comparação dos resultados obtidos pela análise dos distintos corpus e representação do continuum fala/escrita ligado às frequências de uso das nominalizações. 


\section{RESULTADOS}

Segue, na Tabela 1, a síntese dos resultados obtidos pela análise pela frequência de ocorrência dos padrões de nominalização em competição contidos nos textos 1, 2 e 3 que compõem a amostra da pesquisa.

\begin{tabular}{|c|c|c|c|c|c|c|}
\hline \multirow{2}{*}{ Sufixo } & \multicolumn{3}{|c|}{$\begin{array}{c}\text { Frequência } \\
\text { total por sufixo }\end{array}$} & \multicolumn{3}{c|}{$\begin{array}{c}\text { Frequência relativa do emprego do } \\
\text { sufixo/Total de ocorrência de sufixos }\end{array}$} \\
\hline & T1 & T2 & T3 & T1 & T2 & T3 \\
\hline -ção & 109 & 71 & 98 & $66,87 \%$ & $63,39 \%$ & $78,4 \%$ \\
\hline -mento & 12 & 34 & 0 & $7,36 \%$ & $30,35 \%$ & $0 \%$ \\
\hline -ncia & 42 & 3 & 26 & $25,76 \%$ & $2,67 \%$ & $20,8 \%$ \\
\hline -agem & 0 & 4 & 1 & $0 \%$ & $3,57 \%$ & $0,80 \%$ \\
\hline
\end{tabular}

Tabela 1: Síntese da frequência de uso dos sufixos nominalizadores em análise nos artigos (T1 e T2) e no corpus da aula (T3).

Na fala, os resultados da análise das frequências associadas às palavras contidas no T3 apontam a predominância de nominalizações em -ção, seguida, em ordem decrescente de frequência das nominalizações em [-ncia] e [-agem], e a ausência de nominalizações em [-mento]. (V. Tabela 1). Observe-se que, na escrita, os resultados corroboram a tendência de haver um continuum fala/escrita (cf. MARCHUSCHI, 2004) mesmo em textos científicos, comprovando a hipótese 2 da investigação ora empreendida. 
É de se esperar então a existência de um decréscimo na fala de formas afixais típicas do texto científico, como é confirmado pelos cálculos numéricos.

A partir dessa etapa, foram calculadas as frequências associadas ao Ponto de Transição de Goffman, sintetizadas na Tabela 2 a seguir.

\begin{tabular}{|l|}
\hline Ponto T de Goffman (Texto 1) \\
\hline 30,58456 Ponto T de Goffman \\
\hline Ponto T de Goffman (Texto 2) \\
\hline 25,81064 Ponto T de Goffman \\
\hline Ponto T de Goffman (Texto 3) \\
\hline 31,65976 Ponto T de Goffman \\
\hline
\end{tabular}

Tabela 2: Síntese do Ponto T de Goffman

Foram finalmente projetadas as regiões de concentração de palavras com alta carga semântica para os textos e investigadas as recorrências de padrões de nominalizações, concentrados nessas regiões, conforme as tabelas 3, 4 e 5. 


\begin{tabular}{|l|c|c|c|}
\hline \multicolumn{4}{|c|}{ Palavras de alto conteúdo semântico } \\
\hline & Freq. & Rank. & C \\
\hline amostras & 54 & 5 & 270 \\
\hline aceitação & 47 & 9 & 423 \\
\hline provadores & 33 & 14 & 462 \\
\hline vinho & 30 & 15 & 450 \\
\hline amostra & 30 & 18 & 540 \\
\hline Preferência & 24 & 19 & 456 \\
\hline vinhos & 19 & 25 & 475 \\
\hline branco & 18 & 28 & 504 \\
\hline consumidores & 17 & 30 & 510 \\
\hline
\end{tabular}

Tabela 3: Resultados da região de Transição de Goffman do artigo 1.

\begin{tabular}{|l|c|c|c|}
\hline \multicolumn{4}{|c|}{ Palavras de alto conteúdo semântico } \\
\hline & Freq. & Rank. & C \\
\hline bolo & 56 & 8 & 448 \\
\hline bolos & 41 & 14 & 574 \\
\hline farinha & 39 & 16 & 624 \\
\hline fibra & 30 & 22 & 660 \\
\hline controle & 19 & 31 & 589 \\
\hline teste & 17 & 33 & 561 \\
\hline provadores & 17 & 34 & 578 \\
\hline vinhos & 16 & 22 & 352 \\
\hline
\end{tabular}

Tabela 4: Resultados da região de Transição de Goffman do artigo 2.

\begin{tabular}{|l|c|c|c|}
\hline \multicolumn{4}{|c|}{ Palavras de alto conteúdo semântico } \\
\hline & Freq. & Rank. & C \\
\hline produto & 58 & 16 & 928 \\
\hline solubilidade & 28 & 34 & 952 \\
\hline concentração & 27 & 37 & 999 \\
\hline constante & 23 & 44 & 1012 \\
\hline solução & 21 & 56 & 1176 \\
\hline
\end{tabular}

Tabela 5: Resultados da região de Transição de Goffman do artigo 3. 
Todas as tabelas confirmam a predominância de nominalizações em [-ção] no campo semântico da Química Geral e da Vinicultura, área altamente especializada. É de se supor que outras áreas estritamente científicas apresentem padrão semelhante.

Destaque-se de novo que, na fala, os resultados apontam a predominância de nominalizações em -ção, seguida, em ordem decrescente de frequência de uso das nominalizações em [ncia] e [-agem], e pela ausência de nominalizações em -mento. Já na escrita, ocorre a predominância de nominalizações em [-ção], seguida de [-mento], [-ncia] e [-agem].

\section{CONSIDERAÇÕES FINAIS}

A análise da frequência de uso de nominalizações nos artigos permite afirmar que o modelo $[X] \vee \rightarrow[$ X] v -ção ] N é predominante nos corpora em análise. Em contrapartida, verifica-se baixa frequência de uso do padrão $[\mathrm{X}] \mathrm{v} \rightarrow[\mathrm{X}]$ v agem] $\mathrm{N}$, tendendo a zero no T1 e T3. Esses dois padrões descritos de nominalização parecem situar-se em pontos polarizados em um continuum projetado, marcado por uma gradiente de produtividade de formação de nominalizações deverbais na fala e na escrita.

A confrontação dos resultados de análise dos artigos sobre vinicultura com os da aula sobre Química Geral revela 
diferença significativa nas frequências de uso, associadas aos sufixos [-ção] e [-mento] e [-ncia]. Os textos (T1 e T2) sobre Vinicultura apresentaram menor diferença entre as frequências relativas de uso dos sufixos nominalizadores [-ção] e [-mento]. Por comparação, o corpus relativo à aula sobre Química Geral (T3) apresentou maior frequência de uso dos sufixos [-encia] do que no texto 2 (T2) sobre Vinicultura.

Para os corpora analisados, a delimitação da Região de Transição e a observação dos itens lexicais frequentes na Região, na totalidade dos textos, indicou a ocorrência de palavras de alta carga semântica. A investigação das frequências de uso de nominalizações deverbais na Região revelou-se também satisfatória em quase toda a amostra. Constata-se a predominância da função denominadora dos processos analisados, que resultam na constituição de rótulos com representação conceptual nos subcampos de conhecimento.

Assim, esse estudo vem fortalecer trabalhos empíricos similares (cf. Basílio, 1979, 2007; Guedes, 2010; Guedes, Mollica \& Leal, 2011; Guedes, Barbosa \& Santos, 2012) com contribuição a Ciência da Informação. Oferece especial interesse no que tange ao desenvolvimento de sistemas precisos de indexação e recuperação da informação, a partir da aproximação teórica e descritiva entre a teoria lexical 
(cf. ARONOFFF.1976, 1994; ARONOFF \& ANSHEN, 2001) nas Ciências da Linguagem, aliando-se a área de Organização do Conhecimento na $\mathrm{Cl}$.

A análise da frequência de uso de nominalizações em T1, em T2 e em T3, permite afirmar que o modelo [X] $v \rightarrow$ [[X] v -ção] $\mathrm{N}$ é predominante no corpora em análise. Em contrapartida, verifica-se baixa frequência de uso do padrão $[X] \vee \rightarrow[[X] \vee$-agem] $N$, tendendo a zero especialmente em T1. Esses dois padrões descritos de nominalização parecem situar-se em pontos polarizados em um continuum projetado, marcado por um gradiente de produtividade de formação de nominalizações deverbais na fala e na escrita.

A confrontação dos resultados de análise dos artigos em tela com os textos de aula sobre revela diferença significativa nas frequências de uso, associadas ao emprego das formais sufixais [-ção] e [-mento] e [-ncia]. Os textos sobre Vinicultura apresentaram menor diferença entre as frequências relativas ao uso dos sufixos nominalizadores [-ção] e [-mento] . Enquanto que a amostra por da aula de Química Geral no Projeto NURC apresentou maior frequência de uso dos sufixos [-ncia].

Para os corpora analisados, a delimitação da Região de Transição e a observação dos itens lexicais frequentes na 
Região, em um percentual estimado em 100\% dos textos, indicou a ocorrência de palavras de alta carga semântica. A investigação das frequências de uso de nominalizações deverbais na Região revelou-se satisfatória na maioria das amostras examinadas.

Por fim, cabe ressaltar que a investigação desenvolvida revela efetividade da função denominadora dos processos analisados, que resultam na constituição de rótulos com representação conceitual nos subcampos de conhecimento.

As construções em tela podem ser consideradas como estratégia de polidez, na medida em que atendem à adequação de padrões estabelecidos para a escrita cientifica, como propões conforme propõe Van Djick (2012) na interação discurso/e contexto. Mais recentemente defende o autor a relação dos usos e de processos cognitivos das representações mentais da linguagem (Van Dijk, 2014).

\section{REFERÊNCIAS}

Bakhtin, M. M. (1992). Os gêneros do discurso. In: M. M. Bakhtin. Estética da criação verbal. São Paulo: Martins Fontes.

Barbosa, M.F.S.O. (2010). (Im)polidez em EAD. Tese (Doutorado em Linguística) - Programa de Pós-Graduação em Linguística. Universidade Federal do Rio de Janeiro.

Basílio, M. M. P. (2007). Teoria Lexical. 8ed. São Paulo: Ática. (Série Princípios, 88). 
Bazerman, C. (2006). Gêneros textuais, tipificação e interação. São Paulo: Editora Cortez.

Brown, P. \& Levinson, S. (1978-1987). Politeness: some universal in language. Cambridge: Cambridge University Press.

Dahlberg, I. (2006). “Knowledge organization: a new science?" In Knowledge Organization, 33, 1, p.11.

EGGINS, S. (2004). An introduction to systemic functional linguistics. (2nd. ed.). Nova York: Continuum International Publishing Group.

Gil-Salom, L. \& Soler-Monreal C. (2009). Interacting with the reader: politeness strategies in engineering research article discussions. International Journal of English Studies, Special Issue, 175-189.

Goffman, E. (1967). Footing. In: Ribeiro, B.T. 7 Garcez, P.M. (orgs.). Sociolinguística interacional. 2ed. São Paulo: Loyola.

Guedes, V. L. S. (2010). Nominalizações deverbais em artigos científicos: uma contribuição para a análise e a indexação temática da informação. Tese (Doutorado em Linguística) - Programa de Pós-Graduação em Linguística. Universidade Federal do Rio de Janeiro.

Guedes, V. L. S.; Barbosa, M. F. S. O. \& Santos, M. J. V. C. (2012). Recorrência de nominalizações deverbais em resumos de cartas científicas em língua portuguesa como estratégia linguística de polidez. In: Congresso Internacional Interdisciplinar Cortesia: olhares e (re)invenções. Lisboa, Portugal: Universidade Aberta.

Hjørland, B. \& Albrechtsen, H. (1995). Toward a new horizon in information science: Domain-analysis. Journal of the American Society for Information Science, 48, 6, 400-425.

Hjørland, B. (2002). Domain analysis in information science: eleven approaches-traditional as well as innovative. Journal of documentation, $58,4,422-462$.

Hyland, K. (2009). Academic discourse: English in a global context. New York: Continuum International Publishing Group.

Hyland, K. \& Salenger-Meyer, F. (2008). Scientific writing. In: B. Cronin 
(Ed.).Annual Review of Information Science and Technology, 42, 1, 297338.

$\emptyset$ rom, A. (2003). Knowledge Organization in the domain of Art Studies - history, transition and conceptual changes. Knowledge Organization, v.30, n.3-4, 2003.

Pao, M. L. (1989). Concepts of information retrieval. Englewood: Libraries Unlimited, Inc.

Soergel, D., Lauser, B., Liang, A., Fisseha, F., Keizer, J. e Katz, S. (2004). Journal of Digital Information, 4.

SWALES, J. M. (1990). Genre analysis: English in academic and research settings. Cambridge: Cambridge University Press.

ZIMAN, J. M. (1969). Information, communication and knowledge, Nature, 224, 5217, p. 318-324.

ZIPF, G.K. (1949).Human behavior and the Principle of Least Effort. Cambridge, MA: Addison-Wesley.

\section{OUTRAS FONTES}

Andersen, J. (2008). The concept of genre in information studies. Annual Review of Information Science and Technology, 42, Issue 1, p. 339-367. Disponível em: http://onlinelibrary.wiley.com/doi/10.1002/ aris.2008.1440420115/abstract;jsessionid $=2615869$ C6CC4667435FB8A3 B88C5C565.d02t04?deniedAccessCustomisedMessage $=$ \&userlsAuthenti cated $=$ false.

Bazerman, C. (1998). Shaping written Knowledge. The genre and activity of the experimental article in science. Madison, Wisconsin: The University of Wisconsin Press. Disponível em: http://wac.colostate.edu/books/ bazerman_shaping/shaping.pdf Acesso em: 27/05/2013.

Méndez Rodríguez \& Moreiro González (1999). Lenguaje natural e indización automatizada, Ciencias de la Información, 30, 3, p. 11-24. Disponível em: http://www.bib.uc3m.es/ mendez/publicaciones/ articulos/indizacion99.pdf Acesso em: 27/05/2013. 
Okubo, Y. (1997). Bibliometric indicators and analysis of research systems: methods and examples. Paris: OECD Publishing, 1997. Science, Technology and Industry Working Papers, 1997/01. Disponivel em: http://www.oecd. org/officialdocuments/publicdisplaydocumentpdf/?cote=OCDE/GD(9)4 1\&docLanguage=En Acesso em: 27/03/2012.

Pao, M. L. (1978). Automatic text analysis based on transition phenomena of word occurrences. Journal of the American Society for Information Science, New York, v. 29, n.3, p. 121-124, May. Disponível em: http:// onlinelibrary.wiley.com/doi/10.1002/asi.4630290303/pdf Acesso em: 27/03/2012.

Maria Cecilia Mollica. Professora Titular de Linguística da Faculdade de Letras - Depto Linguística E Filologia - UFRJ - 40h/ $\mathrm{DE}$ ). Pesquisadora do CNPq - Nível 1. Pós-doutora em Linguística (UnB, 2012); Doutora em Linguística e Filologia (UFRJ, 1989), Mestra em Letras (PUC-RIO, 1977). Bolsista do Nosso Estado da FAPERJ. Coorda do Mestrado Profissional PROFLETRAS POLO/UFRJ. 5) Líder do Grupo de Pesquisa do PEUL. 6) Líder de Pesquisa do Projeto de Alfabetização da Pró-Reitoria de Extensão da UFRJ (2003-2012). 8) Diretora de Extensão da Faculdade de Letras (2000-2003); 9) Diretora da Faculdade de Letras da UFRJ (2002-2005). 10) Presidente da ABRALIN (2001-2003) contato: ceciliamollica@terra.com.br

Maria de Fatima Sousa de Oliveira Barbosa. Professora Adjunta da UFRJ. Pós-doutoranda pelo IBICT/UFRJ/CAPES. Doutora em Linguística (UFRJ, 2010). Mestre em Tecnologia Educacional nas Ciências da Saúde (UFRJ, 2004). Especialista em Multimídia para Educação a Distância pela Ryerson University - CA (1998), em Informática Educativa pela UERJ (1997) e em Gestão da Informação pelo INT/IBICT/UFRJ (1999). Coordenadora interina/tutora de Língua Portuguesa no curso de Pedagogia a Distância/CEDERJ/UNIRIO. Contato: fatimma.barbosa@gmail.com

Vânia Lisboa da Silveira Guedes Professora Adjunta da FACC/ UFRJ e do PROFLETRAS Polo/UFRJ. Em estágio pós-doutoral no Programa de Pós-graduação em Linguística (PPGL/UFRJ). Doutora 
em Linguística pelo PPGL/UFRJ (2010). Mestre em Ciência da Informação (Cl) pela ECO/UFRJ (1992). Atua na área de Organização do Conhecimento e Bibliometria na $\mathrm{Cl}$, assim como na Análise Crítica do Discurso Científico e Análise Léxico-morfológica na Sociolinguística. Contato: vanialisboa@facc.ufrj.br 